\title{
Morphologic Variants of the Hand Motor Cortex in Developing Brains from Neonates through Childhood Assessed by MR Imaging
}

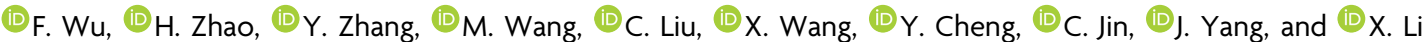

\begin{abstract}
BACKGROUND AND PURPOSE: Knowledge of anatomic markers of the hand motor cortex is essential in the evaluation and treatment of motor neurologic diseases for both adults and developing populations. However, hand motor cortex variants in developing brains remain to be investigated. Our objective was to observe morphologic variants of the hand motor cortex in developing brains from neonates through childhood.
\end{abstract}

MATERIALS AND METHODS: In this study, 542 participants (0 15 years of age) were retrospectively enrolled and divided into different age groups. The hand motor cortex morphology was evaluated on the basis of 3D TWWI. Variations in hand motor cortex variants were compared among different age groups. Inter-gender and interhemispheric differences of hand motor cortex variants were also evaluated.

RESULTS: Various hand motor cortex variants could be observed in developing brains, even in the neonatal period. One new morphologic shape, "immature $\Omega$," was found in neonates and infants. The proportion of this new shape decreased dramatically during the first year after birth, then disappeared after 1 year of age. It persisted for a longer time in the right hemisphere and in males. However, sex or hemispheric effects on the distribution of the proportion of variants were not statistically significant. Furthermore, the proportion of concordance of the bilateral hand motor cortex showed an increasing trend with age $(P=.006)$, higher in females than males.

CONCLUSIONS: Various hand motor cortex variants already existed at birth. The distribution of proportions of different variants developmentally varied during the first year after birth and became stable after 1 year of age. The concordance of the bilateral hand motor cortex could be influenced by age and sex.

ABBREVIATIONS: $\mathrm{ACPC}=$ anterior/posterior commissure; $\mathrm{GA}=$ gestational age; $\mathrm{HMC}=$ hand motor cortex; $\mathrm{PMA}=$ postmenstrual age

$\mathbf{T}$ he hand motor cortex (HMC) has been identified as a knob on the precentral gyrus. ${ }^{1}$ It is always the recognition and stimulation/treatment target in the evaluation and treatment of neurologic motor diseases (eg, stroke, ${ }^{2-6}$ focal hand dystonia, ${ }^{7}$ and amyotrophic lateral sclerosis ${ }^{8}$ ) or glioma. ${ }^{9}$ Furthermore, it is

Received July 6, 2021; accepted after revision October 20.

From the Department of Radiology (F.W., H.Z., Y.Z., M.W., C.L., X.W., Y.C., C.J., J.Y.,

X.L.), the First Affiliated Hospital of Xi'an Jiaotong University, Xi'an, China; and

Department of Radiology (F.W.), Guangzhou Women and Children's Medical

Center, Guangzhou Medical University, Guangzhou, China.

Fan Wu and Huifang Zhao contributed equally to this work.

Jian Yang and Xianjun Li served as senior authors.

This work was supported by the National Natural Science Foundation of China (81971581, 81901823, 81771810, and 81901516) and the Innovation Team Project of Natural Science Fund of Shaanxi Province (2019TD-018).

Please address correspondence to Xianjun Li, PhD, Department of Radiology, the First Affiliated Hospital of Xi'an Jiaotong University, No. 277 West Yanta Road, Xi'an 710061, Shaanxi, China; e-mail: xianj.li@mail.xjtu.edu.cn

- Indicates open access to non-subscribers at www.ajnr.org

Indicates article with online supplemental data.

http://dx.doi.org/10.3174/ajnr.A7386 an identifiable neuroanatomic structure for delineating the corticospinal tract. ${ }^{10}$ Therefore, the identification of the HMC is essentially important for the exploration of the mechanism of handedness, ${ }^{11}$ hand motor skills, ${ }^{12}$ and eye-hand coordination. ${ }^{13}$

MR imaging provides a nonradiative tool for investigating the morphology of the HMC in vivo. The previous known shape of the HMC is a typical hook ${ }^{14,15}$ on the MR imaging sagittal plane, and a V-shaped anatomic signature ${ }^{16}$ and an $\Omega$ on the axial plane. ${ }^{1}$ The $\Omega$ sign was gradually recognized as a reliable landmark for the localization of the HMC. ${ }^{16}$ Furthermore, 5 morphologic variants $(\Omega$, medially asymmetric $\varepsilon, \varepsilon$, laterally asymmetric $\varepsilon$, and null) of the HMC have been recognized on MR imaging in adults. ${ }^{15}$ Knowledge of anatomic markers of the HMC on MR imaging is essential in research and clinical practice, not only for adults but also for the developing population. Specifically, the treatment target of transcranial magnetic stimulation was always placed on the primary motor cortex in children with spastic cerebral palsy with hand dysfunction. ${ }^{17,18}$ For patients with brain tumors, the width and height of the HMC and the distance from the tumor to the HMC on MR imaging are related to neurologic motor deficits. ${ }^{9}$ Although HMC variants have been 
recognized in adults, ${ }^{15}$ the morphology of the HMC in children may be different from that in adults because the brain develops dramatically during early childhood. ${ }^{19,20}$ However, little information has been reported about the characteristics of the HMC on the developing population, and the effects of age and sex on HMC morphology remain to be assessed. Therefore, it is necessary to investigate the morphology of the HMC in developing brains.

On the basis of the above considerations, this study tried to use MR imaging to investigate the morphologic variants of the HMC in developing brains from neonates through childhood. HMC variants were compared among different age groups. Sex and hemisphere effects on HMC variants were also evaluated. Furthermore, the concordance of the bilateral HMC was analyzed.

\section{MATERIALS AND METHODS}

This retrospective study was approved by the institutional review board of the First Affiliated Hospital of Xi'an Jiaotong University. Written informed consent was obtained from parents or guardians of participants.

\section{Subjects}

Subjects were enrolled consecutively from August 2012 to December 2019. According to the postnatal age at MR imaging, the enrolled subjects were divided into neonates (age, $\leq 28$ days), infants ( $>28$ days to $<1$ year), toddlers ( 1 to $<3$ years), preschool children ( 3 to $<6$ years), school-age children ( 6 to $<10$ years), and adolescents (10 to $<15$ years). To reveal more detailed alterations during the neonatal period, we divided neonates into preterm (gestational age [GA], <37 weeks) and term (GA, $\geq 37$ weeks) groups. All infants, toddlers, preschool children, school-age children, and adolescents were term birth. Because of the difference in the disease spectrum between the neonate group and other age groups, we performed the inclusion and exclusion using different criteria.

The detailed inclusion and exclusion criteria were as follows:

Inclusion criteria: neonate group: age at MR imaging, $\leq$ 28 days. Other age groups: 1$) \mathrm{GA} \geq 37$ weeks; 2) age at MR imaging, 29 days $\sim 15$ years of age.

Exclusion criteria: 1) abnormal findings on MR imaging or diseases influencing the brain maturation: neonatal group: punctate white matter lesions, metabolic disorders, intracranial hemorrhage, periventricular leukomalacia, congenital malformations of nervous system, hydrocephalus, small for GA, hypoxic-ischemic encephalopathy, and intracranial infection. Other age groups: focal white matter hyperintensity on T2 FLAIR, enlargement of subarachnoid spaces, dilated Virchow-Robin spaces, encephalomalacia, periventricular leukomalacia, congenital malformations of the nervous system, hydrocephalus, malformation of cerebral vessels, metabolic disorders, and others including cerebral trauma and intracranial tumor, epilepsy, febrile convulsion, tic disorder, visual abnormalities, intracranial infection, hypoxic-ischemic encephalopathy, and mental, behavioral, or neurodevelopmental disorders; 2) incomplete MR images or clinical information (including perinatal characteristics and clinical conditions).

\section{Data Acquisition}

MR images were acquired using a 3T scanner (Signa HDxt; GE Healthcare) with an 8-channel head coil. 3D fast spoiled gradient recalled-echo T1WI was performed using the following parameters: TR/TE, $10.42 / 4.74 \mathrm{~ms}$; isotropic resolution, $1 \times 1 \times 1 \mathrm{~mm}^{3}$; matrix, $240 \times 240$; thickness, $1 \mathrm{~mm}$; FOV, $240 \times 240 \mathrm{~mm}^{2}$.

\section{Image Processing}

The 3D T1WIs of the cerebrum were translated and rotated into the anterior/posterior commissure (ACPC) plane. In adults, the HMC was located at Talairach coordinates of $\mathrm{x}= \pm 34, \mathrm{y}=-29$, $\mathrm{z}=50 .{ }^{15,21}$ Due to the developmental changes of brain morphology, the optimal level for evaluating the HMC was different across individuals. Here, the ACPC plane was defined as the zero point of the z-axis. Then, the evaluation plane above the ACPC line was located at different coordinates in different age groups: neonates, 32 (SD, 3) mm; infants, 39 (SD, 4) mm; toddlers, $46(\mathrm{SD}, 4) \mathrm{mm}$; preschool children, 49 (SD, 4) mm; school-age children, 50 (SD, 3) $\mathrm{mm}$; and adolescents $50(\mathrm{SD}, 3) \mathrm{mm}$.

\section{Morphologic Variant Classification of the HMC}

Five different morphologic variants have been found for the adult HMC: $\Omega$, medially asymmetric $\varepsilon, \varepsilon$, laterally asymmetric $\varepsilon$, and null. ${ }^{15}$ Besides these variants, this work revealed 1 new shape like but different from the $\Omega$ type. Because this shape could be found in neonates and infants and disappeared from the toddler period on, this work termed it "immature $\Omega$ " (Fig 1). The identification of the immature $\Omega$ was performed according to the following appearances: The shape was sloped; the outside angle between any side of the $\mathrm{HMC}$ and the base side was $>90^{\circ}$; the ratio between the height of the HMC and thickness of gray matter was $\geq 4$; and the ratio between the height and width of the HMC was $\leq 1$. For comparison, the $\mathrm{HMC}$ with an outside angle of $\leq 90^{\circ}$, the ratio between the height of the HMC and thickness of gray matter was $\geq 4$, or the $\Omega$ type was classified as the "mature $\Omega$." Two radiologists (with $>5$ years' experience in the interpretation of the pediatric brain MR imaging), blinded to the demographic information of participants, independently evaluated the HMC type, and one of them preformed the evaluation twice. Moreover, disagreements across different evaluations were resolved by discussion with a senior radiologist ( $>15$ years' experience).

\section{Statistical Analysis}

Statistical analyses were performed using SPSS (Version 18; IBM). Two neuroradiologists independently evaluated the HMC. Intra- and interobserver reliability was evaluated using the $\kappa$ test. Categoric variables (the distribution of the HMC variant in different age groups) are shown as percentages and percentages in stacked bar charts. Categoric variables were compared using the Monte Carlo method test. $P<.05$ was considered statistically significant. In multiple comparisons, $P<.0024(.05 / 21)$ was considered statistically significant after the Bonferroni correction. The $\chi^{2}$ test for trend was performed for the proportion of concordance of the bilateral HMC variants in each age group.

\section{RESULTS}

\section{Participants}

According to the inclusion and exclusion criteria, this work enrolled 542 participants, including 78 preterm neonates (40 males), 159 term neonates (103 males), 46 infants (32 males), 49 toddlers (33 


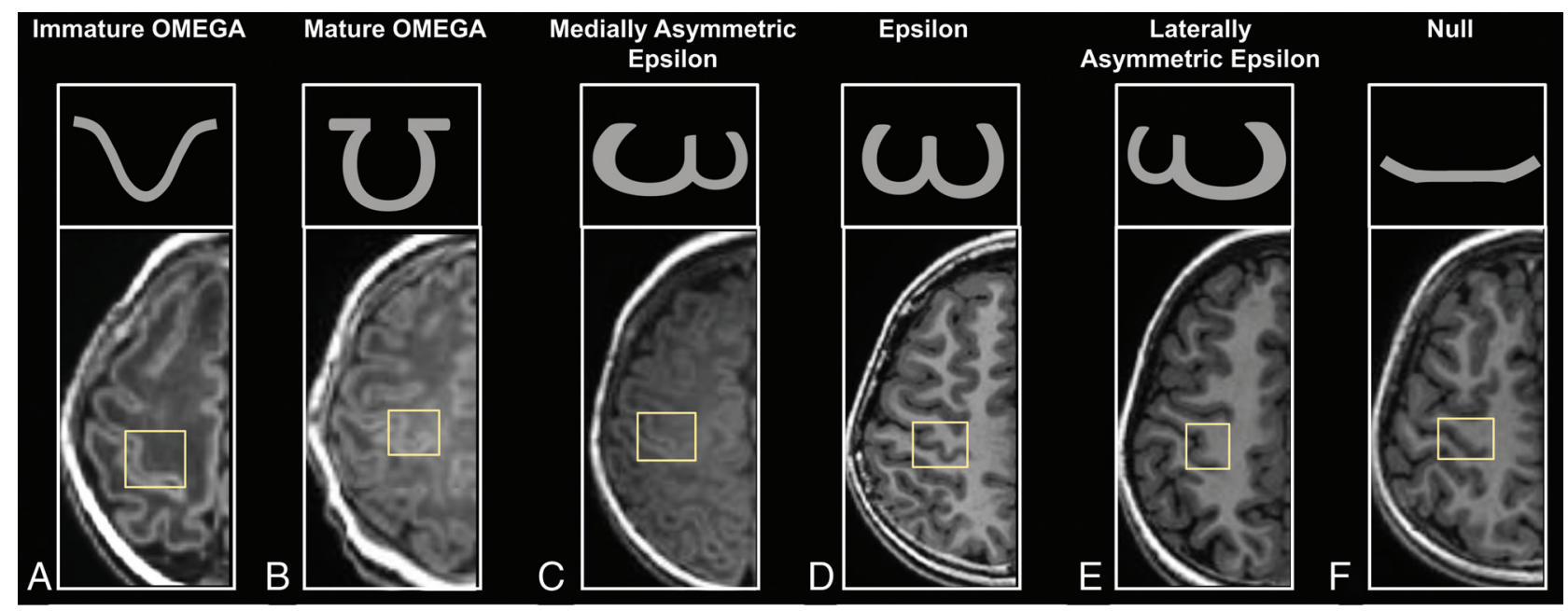

FIG 1. Appearances of HMC variances. Immature $\Omega(A)$ : preterm neonate (female, $\mathrm{GA}=32^{+1}$ weeks, PMA $=32^{+5}$ weeks). Mature $\Omega(B)$ : term neonate (female, $\mathrm{GA}=38^{+2}$ weeks, PMA $=38^{+6}$ weeks). Medially asymmetric $\varepsilon(C)$ : infant (female, $\mathrm{GA}=39^{+1}$ weeks, age $\left.=1.18 \mathrm{months}\right) \cdot \varepsilon(D)$ : adolescent (female, $\mathrm{GA}=42$ weeks, age $=10.36$ years). Laterally asymmetric $\varepsilon(E)$ : school-age children (female, GA $=39^{+6}$ weeks, age $=$ 8.37 years). Null (F): preschool children (male, $\mathrm{GA}=40$ weeks, age $=5.96$ years).

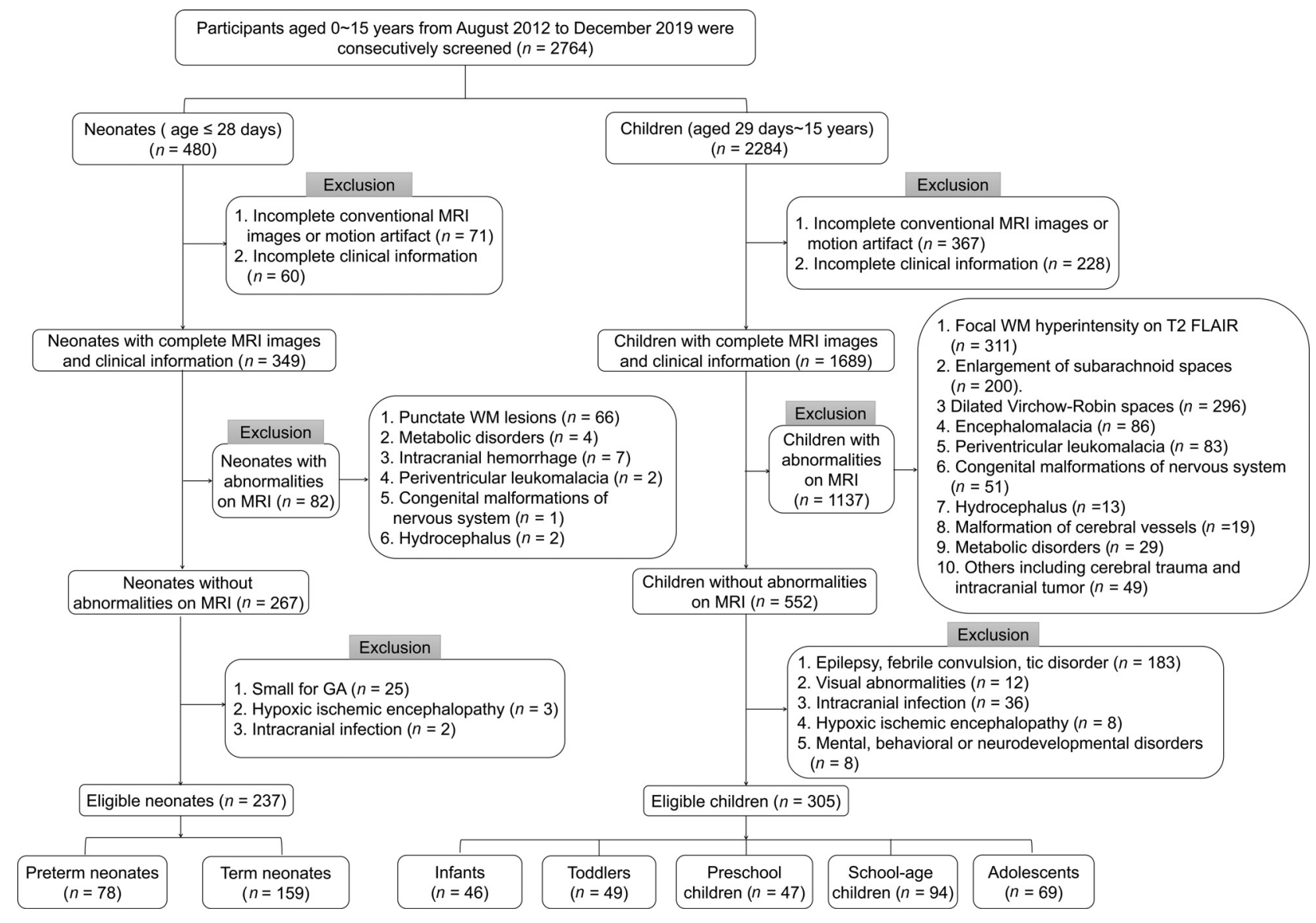

FIG 2. Flow chart of the inclusion and exclusion criteria.

males), 47 preschool children (34 males), 94 school-age children (53 males), and 69 adolescents (41 males) (Fig 2). No significant differences in GA, postnatal age, or postmenstrual age (PMA) at MR imaging were found between males and females in each age group $(P>.05)$.

\section{Intra- and Interobserver Reliability}

The intraobserver and interobserver agreement for the HMCtype classification was $93.91 \%(\kappa$ value $=0.89$; standard error $=0.02)$ and $90.59 \%(\kappa$ value $=0.84$; standard error $=$ $0.02)$, respectively. 


\section{Percentage of Morphologic Variants in Different Age Groups}

In total, 6 variants (including 5 conventional variants and the immature $\Omega$ ) could be found in participants. The sequence of the percentage of 542 participants (1084 HMCs) in descending order was the following: mature $\Omega$ (75.65\%), $\varepsilon$ (14.49\%), immature $\Omega$ (6.46\%), null (2.21\%), laterally asymmetric $\varepsilon(1.11 \%)$, and medially asymmetric $\varepsilon(0.09 \%)$. The proportions of HMC variants in different age groups are shown in Fig 3.

The percentage distribution of HMC variants was statistically different across age groups. In the pair-wise comparison across 7 age groups (Online Supplemental Data), there were significant differences in the distribution of the HMC variant between the preterm neonates and other age groups $(P<.0024)$. The mature $\Omega$ showed the trend of "increasing, relatively stable." Except for neonates, there was no significant difference in the distribution of the HMC variants among other age groups $(P>.0024)$. Specifically, the proportion of the immature $\Omega$ HMC decreased from $25.64 \%$ in preterm neonates to $8.81 \%$ in term neonates, $2.17 \%$ in infants, and $0 \%$ in toddlers. The proportion of the mature $\Omega$ HMC increased from $57.69 \%$ in preterm neonates to $73.58 \%$ in term neonates and $76.09 \%$ in infants and was stable from toddler age on at $76.81 \%$ $88.78 \%$ (median, $83.25 \%$ ). The proportion of the $\varepsilon(9.18 \% \sim$
20.29\%; median, $14.78 \%)$, the laterally asymmetric $\varepsilon(0 \% \sim 3.26 \%$; median, $1.02 \%)$, the medially asymmetric $\varepsilon(0 \% \sim 1.09 \%$; median, $0 \%)$, and the null HMC ( $0 \% \sim 6.41 \%$; median, $1.26 \%)$ did not differ significantly among age groups.

\section{Morphologic Variants of the HMC in Different Sexes and Hemispheres}

As shown in Fig 4, there was no significant difference in the distribution proportion between males and females or between left and right hemispheres in each age group $(P>.05)$. Figure 4 also shows that the immature $\Omega$ sign seems to persist for a longer time in infants in the right hemisphere and in males.

Furthermore, the interhemispheric concordance of the HMC variants was also evaluated at the individual level. As for the same variant between the left and right hemispheres, the $\Omega$ type accounted for $97.50 \%$ (immature $\Omega$ accounted for $25 \%$ ) in preterm neonates, and the null accounted for $2.50 \%$. The $\Omega$ type accounted for $95.05 \%$ (the immature $\Omega$ accounted for $5.94 \%$ ) in term neonates, the $\varepsilon$ accounted for $3.96 \%$, and the lateral $\varepsilon$ accounted for $0.99 \%$. The mature $\Omega$ was also the HMC variant with the maximum proportion in other age groups (accounting for $>90 \%$ ): infants $(90.32 \%)$, toddlers (100\%), preschool children (94.74\%), school-age children (94.12\%), and adolescents (91.30\%). The proportion of the interhemispheric concordance showed an increasing trend with age $\left(\chi^{2}=7.540, P=.006\right)$.

As for different sexes, the ratios of participants with the same bilateral HMC variant were $\geq 50 \%$ in both males and females (Table). The consistency ratio of females was higher than in males in most age groups.

\section{DISCUSSION}

This study demonstrated morphologic variants of the HMC in developing brains from neonates through childhood. Besides the previously proposed 5 variants of the HMC in adults, ${ }^{1,15}$ we classified the $\Omega$ into 2 subtypes: mature $\Omega$ and immature $\Omega$. Results

FIG 3. The proportional distribution of HMC variants in different age groups.

Right

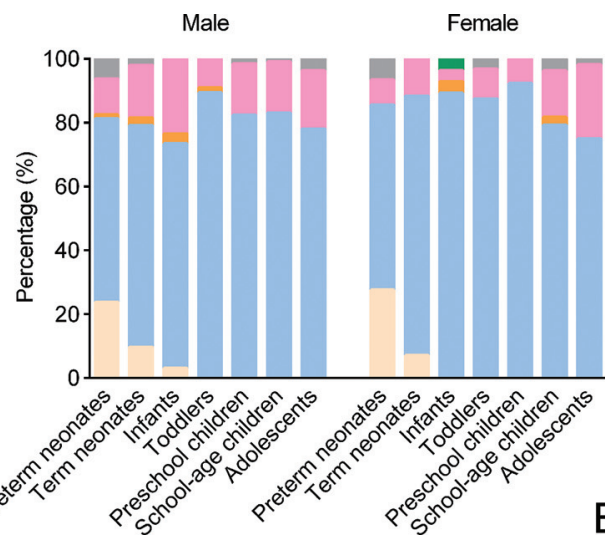

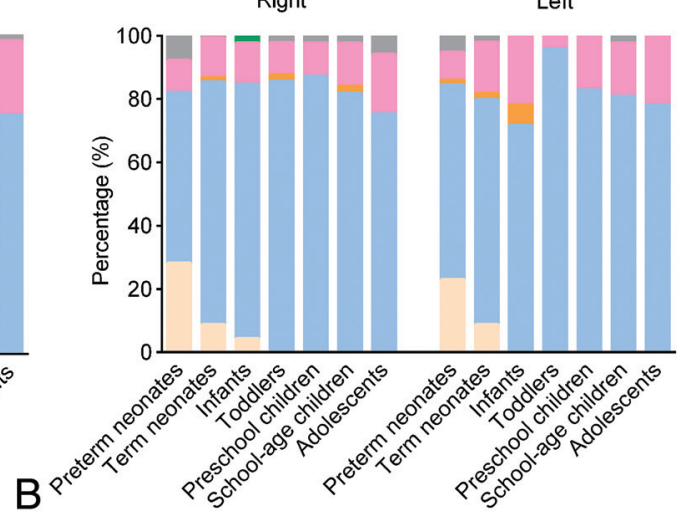

Null

Medially asymmetric epsilon Epsilon

Laterally asymmetric epsilon

Mature omega

Immature omega

FIG 4. The proportional distribution of HMC variants in different sexes $(A)$ and different hemispheres (B). 
The proportion of concordance of the HMC variant in bilateral hemispheres in males and females of different age groups

\begin{tabular}{lccc}
\hline \multicolumn{1}{c}{ Age Stage } & Male (\%) & Female (\%) & Total (\%) \\
\hline Preterm neonates & 52.50 & 50.00 & 51.28 \\
Term neonates & 60.19 & 69.64 & 63.52 \\
Infants & 62.50 & 78.57 & 67.39 \\
Toddlers & 78.79 & 75.00 & 77.55 \\
Preschool children & 79.41 & 84.62 & 80.85 \\
School-age children & 69.81 & 75.61 & 72.34 \\
Adolescents & 65.85 & 67.86 & 66.67 \\
Average & 67.01 & 71.61 & 68.51 \\
\hline
\end{tabular}

demonstrated that various HMC variants already existed at birth and the proportional distribution of variants changed dramatically during the first year after birth. The concordance of the bilateral HMC showed an increasing trend with age. It could also be influenced by sex. Results here may provide a reference for the future investigations of the influence of pediatric diseases on the HMC.

\section{HMC Morphologic Variants and Brain Development}

Results here revealed that various HMC variants already existed in neonates. The immature $\Omega$ type accounted for a noticeable proportion. Typically, the central sulcus forms at a GA of 20-23 weeks, while the precentral gyrus forms at a GA of 24-27 weeks, and the secondary cerebral gyrus, at a GA of $32-35$ weeks. ${ }^{22,23}$ In this study, the PMA at MR imaging of all neonates was $>32$ weeks. Therefore, the HMC in the immature state could be observed in neonates here. This study also found that the distribution of proportions in different variants of the HMC dramatically changed with age, especially before 1 year of age. The proportion of the immature HMC decreased, while the mature HMC increased. This observation may be because the cortex is still in a continuous folding process. During the third trimester of gestation, cortical folding changes dramatically in the brain. ${ }^{24}$ A previous study observed that the synapse formation and the synapse pruning begin around a GA of 20 weeks and the myelin formation begins around a GA from 30 to 32 weeks. ${ }^{25}$ These observations suggest that changes of the HMC morphology may also be associated with the ongoing myelin sheath and synaptic remodeling processes. ${ }^{20,26}$ It has been proposed that the tension-mediated folding increases as the axons strongly pull the interconnected regions together. ${ }^{27}$ Development of axons underlying the central sulcus may cause the morphology of the HMC to change toward the mature type.

In addition, the cortical surface area of the precentral gyrus continues to increase after 1 year of age, ${ }^{20,28}$ while the sulci depth remains stable. ${ }^{29}$ Therefore, the change of the precentral gyrus after 1 year of age here mainly reflects the increase of the gyrus width. Meanwhile, spatial distributions of the sulcal pits (the locally deepest points in sulci) show high consistency between infants and adults. ${ }^{30}$ In agreement with these cortical maturation characteristics, the proportional distribution in the HMC variants reaches a relatively stable state after 1 year of age.

\section{Potential Influencing Factors on HMC Morphologic Variants}

HMC variants in adults have demonstrated that the proportions of sequences from high to low are as follows: $\Omega, \varepsilon$, laterally asymmetric $\varepsilon$, medially asymmetric $\varepsilon$, and null. ${ }^{15}$ In this study, the distribution of the mature $\Omega$ type in adolescents was similar to that of adults. Meanwhile, there were also differences: The ratio of $\varepsilon$ in the current work was higher than that of adults, and the ratios of laterally asymmetric $\varepsilon$ and medially asymmetric $\varepsilon$ were lower. This finding may be due to a combination of factors of ethnic, genetic, customary, and environmental differences during crucial developmental stages of the brain. Previous studies have shown that the primary motor cortex in higher primates has internal subdivisions in the rostrocaudal direction and in 2 sectors connected to the spinal cord. ${ }^{31,32}$

Additionally, in a direct electrophysiologic study of humans, the anatomo-functional subdivisions of the HMC include $2 \mathrm{sec}$ tors: the caudal one and the rostral one, playing different roles in motor control. ${ }^{33}$ The caudal HMC is the most excitable sector. The rostral HMC is a crucial area for shaping functional synergies for hand-object interaction. These findings suggest that the human hand knob is an anatomo-functional heterogeneous region organized along a motor-cognitive gradient. ${ }^{34}$ Thus, we speculated that the position of the middle fissure of $\varepsilon$ in morphology may be related to these 2 sectors in the anatomo-functional subdivision. In families of the enrolled participants, early fine-motor skills of manipulating chopsticks and writing implements are expected. ${ }^{35}$ Exuberant connectivity of the brain in the early life is pruned by competition, influenced by the early experience. ${ }^{19}$ Thus, plasticity and adaptation of the early brain development could lead to differences in the HMC morphology, especially in $\varepsilon$, laterally asymmetric $\varepsilon$, and medially asymmetric $\varepsilon .^{19}$

The proportion of the same HMC variant in the bilateral hemispheres was higher than that of the inconsistent HMC variants in the bilateral hemispheres. According to comparisons across preterm neonates, term neonates, infants, and toddlers, age was a potential factor influencing the concordance of the HMC variant in the bilateral hemispheres. This finding may be related to the maturation of HMC morphology. With the process of brain development and cortex folding, some of the HMC changed from the immature $\Omega$ type into the mature $\Omega$ type. This feature may be the main factor leading to the increase of the concordance of the HMC variant in the bilateral hemispheres, especially during the first year after birth. Moreover, the immature $\Omega$ sign seems to persist for a longer time on the right side. This interhemispheric difference may be associated with the lateralization of motor function during development. A previous study indicated that early brain lateralization in humans could be influenced or reinforced by the asymmetric posture of the developing fetus. ${ }^{36}$ From 14 weeks after conception, fetuses preferentially turn their heads to the right and suck on their right thumbs; this preference appears to be maintained throughout pregnancy. ${ }^{37}$ In addition, most fetuses are in a head-down orientation on the mother's left side, a preferred position during the final trimester, favoring movement of the right arm by providing more space on the right side, thus contributing to the hand motor lateralization. ${ }^{36,38}$ Thus, early increased movement of the right hand may promote the folding and maturation of the left hemisphere HMC. Meanwhile, the neonatal left precentral gyrus 
has more efficient communications than the right homolog. ${ }^{39}$ After delivery and when in a supine position, most neonates prefer to lie with their heads turned to the right, resulting in greater perceptual experience related to the right hand. ${ }^{40}$ Therefore, a shorter persisting time of the immature $\Omega$ type in the left hemisphere may be partly a result of the adaption to lateralized functional needs at birth.

Furthermore, the proportion of consistent HMC variants in the bilateral hemispheres was higher in females than that in males. According to a previous study, an interhemispheric concordance for HMC variants was observed only in females. ${ }^{15}$ Several studies on brain asymmetry suggested that the male brain may be more lateralized or asymmetric than the female brain. ${ }^{41-43}$ In an animal study, it was also observed that preventing the flow of androgens from the testis to the brain blocks the formation of the normal rightward brain asymmetry in male rats. Similarly, the female pattern can be reversed to the male pattern by neonatal ovariectomy. ${ }^{44}$

These findings suggest that levels of androgenic and ovarian steroids play a part in regulating brain asymmetry. The growth of children is accompanied by changes in the levels of endocrine hormones, including sex hormones in females and males. ${ }^{45}$ Therefore, sex may be another potential influencing factor on the concordance of the HMC variant in the bilateral hemispheres. Moreover, it has been found that males have smaller fractional anisotropy and larger axial diffusivity than females from birth to 2 years of age. ${ }^{46}$ This finding suggests that the motor tract development in males is slightly behind that in females. ${ }^{46}$ Meanwhile, the tension-mediated folding increases as axons strongly pull the interconnected regions together. ${ }^{27}$ Therefore, a longer persisting time of the immature $\Omega$ type in males may be partly a result of the inter-gender difference in the motor-related white matter maturation.

\section{Limitations}

There are several limitations. First, although we included a large sample of subjects $0-15$ years of age, samples were from 1 single center. Moreover, not all participants were recruited from healthy families. Participants were enrolled partly from the those with healthy findings on examinations and subjects with clinical symptoms (details are listed in Online Supplemental Data). There may be selection bias, though no subjects had abnormalities on MR imaging. Multisite research is necessary in the future to further verify our results. Second, changes in the morphology of individuals were not followed up. Therefore, this was a cross-sectional study. Changes in the variance were solely statistical associations rather than longitudinal observations. Third, this study did not assess the HMC morphology quantitatively. The relationship between morphologic parameters of the HMC and hand function outcomes remains to be investigated in further work.

\section{CONCLUSIONS}

This study demonstrated that various HMC variants already existed at birth. The distribution of proportions of different variants developmentally varied during the first year after birth and became stable after 1 year of age. The concordance of the bilateral HMC could be influenced by age and sex.

Disclosure forms provided by the authors are available with the full text and PDF of this article at www.ajnr.org.

\section{REFERENCES}

1. Yousry TA, Schmid UD, Alkadhi H, et al. Localization of the motor hand area to a knob on the precentral gyrus: a new landmark. Brain 1997;120:141-57 CrossRef Medline

2. Butefisch CM, Kleiser R, Korber B, et al. Recruitment of contralesional motor cortex in stroke patients with recovery of hand function. Neurology 2005;64:1067-69 CrossRef Medline

3. Dong Y, Dobkin BH, Cen SY, et al. Motor cortex activation during treatment may predict therapeutic gains in paretic hand function after stroke. Stroke 2006;37:1552-55 CrossRef Medline

4. Zimerman M, Heise KF, Hoppe J, et al. Modulation of training by single-session transcranial direct current stimulation to the intact motor cortex enhances motor skill acquisition of the paretic hand. Stroke 2012;43:2185-91 CrossRef Medline

5. Laible M, Grieshammer S, Seidel G, et al. Association of activity changes in the primary sensory cortex with successful motor rehabilitation of the hand following stroke. Neurorehabil Neural Repair 2012;26:881-88 CrossRef Medline

6. Revill KP, Haut MW, Belagaje SR, et al. Hebbian-type primary motor cortex stimulation: a potential treatment of impaired hand function in chronic stroke patients. Neurorehabil Neural Repair 2020;34:159-71 CrossRef Medline

7. Kimberley TJ, Pickett KA. Differential activation in the primary motor cortex during individual digit movement in focal hand dystonia vs. healthy. Restor Neurol Neurosci 2012;30:247-54 CrossRef Medline

8. Wang Y, Li X, Chen W, et al. Detecting neuronal dysfunction of hand motor cortex in ALS: a MRSI study. Somatosens Mot Res 2017;34:15-20 CrossRef Medline

9. Jingshan L, Shengyu F, Xing F, et al. Morphometry of the hand knob region and motor function change in eloquent area glioma patients. Clin Neuroradiol 2019;29:243-51 CrossRef Medline

10. Dalamagkas $K$, Tsintou $M$, Rathi $Y$, et al. Individual variations of the human corticospinal tract and its hand-related motor fibers using diffusion MRI tractography. Brain Imaging Behav 2020;14:696-714 CrossRef Medline

11. van den Berg FE, Swinnen SP, Wenderoth N. Involvement of the primary motor cortex in controlling movements executed with the ipsilateral hand differs between left- and right-handers. $J \operatorname{Cogn}$ Neurosci 2011;23:3456-69 CrossRef Medline

12. Amunts K, Schlaug G, Jäncke L, et al. Motor cortex and hand motor skills: structural compliance in the human brain. Hum Brain Mapp 1997;5:206-15 CrossRef Medline

13. Mathew J, Eusebio A, Danion F. Limited contribution of primary motor cortex in eye-hand coordination: a TMS study. J Neurosci 2017;37:9730-40 CrossRef Medline

14. Salamon G, Martini P, Ternier F, et al. Topographical study of supratentorial brain tumors. J Neuroradiol 1991;18:123-40 Medline

15. Caulo M, Briganti C, Mattei PA, et al. New morphologic variants of the hand motor cortex as seen with MR imaging in a large study population. AJNR Am J Neuroradiol 2007;28:1480-85 CrossRef Medline

16. Puce A, Constable RT, Luby ML, et al. Functional magnetic resonance imaging of sensory and motor cortex: comparison with electrophysiological localization. J Neurosurg 1995;83:262-70 CrossRef Medline

17. Gupta M, Lal Rajak B, Bhatia D, et al. Effect of r-TMS over standard therapy in decreasing muscle tone of spastic cerebral palsy patients. J Med Eng Technol 2016;40:210-16 CrossRef Medline

18. Rajak B, Gupta M, Bhatia D, et al. Effect of repetitive transcranial magnetic stimulation on hand function of spastic cerebral palsy children. Journal of Neurological Disorders 2017;5:1000329 CrossRef 
19. Stiles J, Jernigan TL. The basics of brain development. Neuropsychol Rev 2010;20:327-48 CrossRef Medline

20. Gilmore JH, Knickmeyer RC, Gao W. Imaging structural and functional brain development in early childhood. Nat Rev Neurosci 2018;19:123-37 CrossRef Medline

21. Kido DK, LeMay M, Levinson AW, et al. Computed tomographic localization of the precentral gyrus. Radiology 1980;135:373-77 CrossRef Medline

22. Zilles K, Schleicher A, Langemann C, et al. Quantitative analysis of sulci in the human cerebral cortex: development, regional heterogeneity, gender difference, asymmetry, intersubject variability and cortical architecture. Hum Brain Mapp 1997;5:218-21 CrossRef Medline

23. White T, Su S, Schmidt M, et al. The development of gyrification in childhood and adolescence. Brain Cogn 2010;72:36-45 CrossRef Medline

24. Kim H, Lepage C, Maheshwary R, et al. NEOCIVET: towards accurate morphometry of neonatal gyrification and clinical applications in preterm newborns. Neuroimage 2016;138:28-42 CrossRef Medline

25. Khundrakpam BS, Lewis JD, Zhao L, et al. Brain connectivity in normally developing children and adolescents. Neuroimage 2016;134:192203 CrossRef Medline

26. Blanton RE, Levitt JG, Thompson PM, et al. Mapping cortical asymmetry and complexity patterns in normal children. Psychiatry Res 2001;107:29-43 CrossRef Medline

27. Van Essen DC. A tension-based theory of morphogenesis and compact wiring in the central nervous system. Nature 1997;385:313-18 CrossRef Medline

28. Remer J, Croteau-Chonka E, Dean DC, et al. Quantifying cortical development in typically developing toddlers and young children, 1-6 years of age. Neuroimage 2017;153:246-61 CrossRef Medline

29. Li G, Wang L, Shi F, et al. Construction of $4 \mathrm{D}$ high-definition cortical surface atlases of infants: methods and applications. Med Image Anal 2015;25:22-36 CrossRef Medline

30. Meng Y, Li G, Lin W, et al. Spatial distribution and longitudinal development of deep cortical sulcal landmarks in infants. Neuroimage 2014;100:206-18 CrossRef Medline

31. Rathelot JA, Strick PL. Subdivisions of primary motor cortex based on cortico-motoneuronal cells. Proc Natl Acad Sci U S A 2009;106:918-23 CrossRef Medline

32. Witham CL, Fisher KM, Edgley SA, et al. Corticospinal inputs to primate motoneurons innervating the forelimb from two divisions of primary motor cortex and area 3a. J Neurosci 2016;36:2605-16 CrossRef Medline

33. Vigano L, Fornia L, Rossi M, et al. Anatomo-functional characterisation of the human "hand-knob": a direct electrophysiological study. Cortex 2019;113:239-54 CrossRef Medline

34. Simone L, Viganò L, Fornia L, et al. Distinct functional and structural connectivity of the human hand-knob supported by intraoperative findings. $J$ Neurosci 2021;41:42-43 CrossRef Medline

35. Chow SM, Henderson SE, Barnett AL. The Movement Assessment Battery for Children: a comparison of 4-year-old to 6-year-old children from Hong Kong and the United States. Am J Occup Ther 2001;55:55-61 CrossRef Medline

36. Duboc V, Dufourcq P, Blader P, et al. Asymmetry of the brain: development and implications. Annu Rev Genet 2015;49:647-72 CrossRef Medline

37. Hepper PG, Shahidullah S, White R. Origins of fetal handedness. Nature 1990;347:431 CrossRef Medline

38. Previc FH. A general theory concerning the prenatal origins of cerebral lateralization in humans. Psychol Rev 1991;98:299-334 CrossRef Medline

39. Ratnarajah N, Rifkin-Graboi A, Fortier MV, et al. Structural connectivity asymmetry in the neonatal brain. Neuroimage 2013;75:187-94 CrossRef Medline

40. Michel GF. Right-handedness: a consequence of infant supine head-orientation preference? Science 1981;212:685-87 CrossRef Medline

41. Shaywitz BA, Shaywitz SE, Pugh KR, et al. Sex differences in the functional organization of the brain for language. Nature 1995;373:60709 CrossRef Medline

42. Ruigrok AN, Salimi-Khorshidi G, Lai MC, et al. A meta-analysis of sex differences in human brain structure. Neurosci Biobehav Rev 2014;39:34-50 CrossRef Medline

43. Sowell ER, Peterson BS, Kan E, et al. Sex differences in cortical thickness mapped in $\mathbf{1 7 6}$ healthy individuals between $\mathbf{7}$ and $\mathbf{8 7}$ years of age. Cereb Cortex 2007;17:1550-60 CrossRef Medline

44. Toga AW, Thompson PM. Mapping brain asymmetry. Nat Rev Neurosci 2003;4:37-48 CrossRef Medline

45. Herting MM, Maxwell EC, Irvine C, et al. The impact of sex, puberty, and hormones on white matter microstructure in adolescents. Cereb Cortex 2012;22:1979-92 CrossRef Medline

46. Geng X, Gouttard S, Sharma A, et al. Quantitative tract-based white matter development from birth to age 2 years. Neuroimage 2012;61:542-57 CrossRef Medline 